\title{
APPROXIMATION OF APPROXIMATE FIBRATIONS BY BUNDLE MAPS
}

\author{
Y. H. IM
}

\begin{abstract}
In this paper, we give some conditions under which approximate fibrations can be approximated by locally trivial bundle maps.
\end{abstract}

\section{INTRODUCTION}

Recall that an approximate fibration is a proper map $p: M \rightarrow B$ between locally compact ANRs such that $p$ satisfies the following homotopy property: Given an open cover $\varepsilon$ of $B$, an arbitrary space $X$ and two maps $g: X \rightarrow M$ and $F: X \times I \rightarrow B$ such that $p \circ g=F_{0}$, there exists a map $G: X \times I \rightarrow M$ such that $G_{0}=g$ and $p \circ G$ is $\varepsilon$-close to $F$. The purpose of this paper is to investigate conditions under which approximate fibrations can be approximated by locally trivial bundles.

In [23], L. S. Husch showed that an approximate fibration $p: M^{n} \rightarrow S^{1}$ $(n \geq 6)$ can be approximated by a locally trivial bundle map if and only if $p$ is homotopic to a Hurewicz fibration. Furthermore, he gave an example of a closed manifold $M$ and an approximate fibration $p: M^{n} \rightarrow S^{1} \quad(n \geq 6)$ such that $p$ cannot be approximated by a Hurewicz fibration. T. A. Chapman and S. Ferry [5] obtained the same result for Hilbert cube manifolds. Also they gave an example of an approximate fibration $p: M^{n} \rightarrow S^{2} \quad(n \geq 5)$ which is homotopic to a locally trivial bundle map, but cannot be approximated by a locally trivial bundle map [7]. In [19], R. E. Goad obtained that if $p: M^{n+2} \rightarrow B^{n} \quad(n \geq 6)$ is an approximate fibration between manifolds whose fiber is shape equivalent to the torus $T^{2}$, then $p$ can be approximated by a torus bundle.

Later we extend Goad's result, proving that if $p: M^{n+2} \rightarrow B^{n}(n \geq 3)$ is an approximate fibration between manifolds whose fiber is shape equivalent to a closed orientable surface $F^{2}$ with nonpositive Euler characteristic, then $p$ can be approximated by a locally trivial bundle.

Let $F^{m}$ be a compact manifold. We denote by $S(F)$ the set of equivalence classes of the form $[f]$, where $f: M^{m} \rightarrow F^{m}$ is a homotopy equivalence of a compact manifold $M^{m}$ to $F^{m}$ which is a homeomorphism of $\partial M$ to $\partial F$.

Received by the editors June 4, 1991.

1991 Mathematics Subject Classification. Primary 57N15, 55R65; Secondary 57N25, 57N99.

Key words and phrases. Approximate fibration, locally trivial bundle, structure set, fiber homotopy equivalence, upper semicontinuous decomposition.

This paper is a part of the author's Ph.D. Dissertation written under the direction of Professor Robert J. Daverman at the University of Tennessee. The author wishes to thank Professor Daverman for his support and guidance. 
Another such map $f^{\prime}: M^{\prime} \rightarrow F$ is defined to be equivalent to $f$ provided that there exists a homeomorphism $h: M \rightarrow M^{\prime}$ for which $h \circ f^{\prime} \simeq f$. If $T^{n}$ is the $n$-torus and $e: T^{n} \rightarrow T^{n}$ is any standard finite cover, then there is a transfer map $\hat{e}: S\left(T^{n} \times F\right) \rightarrow S\left(T^{n} \times F\right)$ defined by $\hat{e}([f])=[\tilde{f}]$, where $\tilde{f}$ comes from the pull-back diagram

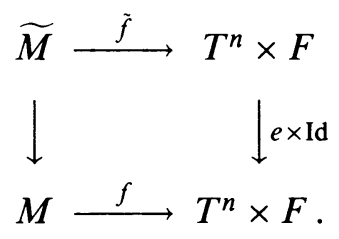

We use $S_{0}\left(T^{n} \times F\right)$ to denote those elements of $S\left(T^{n} \times F\right)$ that are invariant under any of these transfer maps.

In [6], T. A. Chapman gave the following nice results by applying controlled engulfing and torus geometry. These are the key results to determine whether approximate fibrations can be approximated by locally trivial bundle maps.

Theorem 1.1. Let $n \geq 0$ be an integer. For any $\varepsilon>0$, there exists $a \delta>0$ so that if $f: M^{m+n} \rightarrow R^{n} \times F^{m}$ is a $p^{-1}(\delta)$-equivalence for which $f \mid \partial M: \partial M \rightarrow$ $R^{n} \times \partial F^{m}$ is a homeomorphism, where $M^{m+n}$ is a manifold, $F^{m}$ is a compact manifold with boundary and $m+n \geq 5$, then there is an element $\sigma(f)$ of $S_{0}\left(T^{n} \times F\right)$ which vanishes if and only if $f$ is $p^{-1}(\varepsilon)$-homotopic to a homeomorphism ( $p$ denotes the projection onto $R^{n}$ ).

Theorem 1.2. Let $B^{n}$ be a manifold, $\alpha$ be an open cover of $B^{n}$, and $m+n \geq 5$ be an integer. Then there exists an open cover $\beta$ of $B$ so that if $M^{m+n}$ is $a$ manifold, $p: E^{m+n} \rightarrow B^{n}$ is a locally trivial bundle with fiber a compact manifold $F^{n}$, and $f: M \rightarrow E$ is a $p^{-1}(\beta)$-equivalence which is a homeomorphism from $\partial M$ to $\partial E$, then $f$ is $p^{-1}(\alpha)$-homotopic (rel $\partial M$ ) to a homeomorphism provided that $S_{0}\left(T^{i} \times I^{j} \times F\right)$ is zero for $i+j=n$.

By using the above theorems, we have the following main result. For notation, $H$ is the space of self-homeomorphism of $F^{m}$ with the compact-open topology, and $G$ is the space of self-homotopy equivalences of $F^{m}$ with the compactopen topology.

Theorem 1.3. Let $B^{n}$ be an n-dimensional polyhedron, $p: M^{m+n} \rightarrow B^{n}$ be an approximate fibration whose fiber is homotopy equivalent to a closed manifold $F^{m}$, where $M^{m+n}$ is an $(m+n)$-dimensional manifold, and $m+n \geq 5$. Suppose the natural inclusion map $\mathbf{H} \rightarrow \mathbf{G}$ satisfies

(1) $\pi_{i}$-monomorphism for $0 \leq n-2$,

(2) $S_{0}\left(T^{i} \times I^{j} \times F\right)=0$ for $i+j=n$.

Then $p$ can be approximated by a locally trivial bundle map.

For the completion of this paper, we give some applications as a consequence of the main result.

The following notations will be used through this paper. If $\alpha$ is an open cover of $Y$, then a homotopy $h_{t}: X \rightarrow Y$ is an $\alpha$-homotopy provided that each set $\left\{h_{t}(x) \mid 0 \leq t \leq 1\right\}$ lies in some element of $\alpha$.

A proper map $\bar{f}: \bar{X} \rightarrow Y$ is said to be an $\alpha$-equivalence if there is a proper map $g: Y \rightarrow X$ and proper homotopies $\phi_{t}: g \circ f \simeq \operatorname{Id}_{X}, \theta_{t}: f \circ g \simeq \operatorname{Id}_{Y}$ such 
that $f \circ \phi_{t}: X \rightarrow Y$ and $\theta_{t}: Y \rightarrow Y$ are $\alpha$-homotopies. We write this as

$$
\phi_{t}: g \circ f \stackrel{f^{-1}(\alpha)}{\simeq} \text { Id and } \theta_{t}: f \circ g \stackrel{\alpha}{\simeq} \text { Id }
$$

where $f^{-1}(\alpha)$ denotes the open cover of $X$ defined by $f^{-1}(\alpha)=\left\{f^{-1}(U) \mid U \in\right.$ $\alpha\}$.

If $\alpha$ is an open cover, then a proper map $f: X \rightarrow Y$ is said to be an $\alpha$-fibration if for all maps $F: Z \times[0,1] \rightarrow Y$, and $\widetilde{F}_{0}: Z \rightarrow X$ for which $f \circ \widetilde{F}_{0}=F_{0}$, there is a map $G: Z \times[0,1] \rightarrow X$ such that $G_{0}=\widetilde{F}_{0}$ and $f \circ G$ is $\alpha$-close to $F$. This latter statement means that given any $(z, t) \in Z \times[0,1]$, there is an element $U$ of $\alpha$ containing both $f \circ G(z, t)$ and $F(z, t)$. Note that a proper map $f: X \rightarrow Y$ is an approximate fibration provided that it is an $\alpha$-fibration for every open cover $\alpha$ of $Y$.

A map $p: E \rightarrow B$ is called a Hurewicz fibration if $p$ has the following homotopy lifting property with respect to every space $X$ : Given maps $f^{\prime}: X \rightarrow$ $E$ and $F: X \times I \rightarrow B$ such that $F(x, 0)=p \circ f^{\prime}(x)$ for $x \in X$, there is a map $F^{\prime}: X \times I \rightarrow E$ such that $F^{\prime}(x, 0)=f^{\prime}(x)$ for $x \in X$ and $p \circ F^{\prime}=F$.

If $f: E \rightarrow B$ is any map, where $B$ is path connected, then we define

$$
\mathscr{E}=\left\{(e, w) \in E \times B^{I} \mid f(e)=w(0)\right\} .
$$

$\left(B^{I}\right.$ is the space of paths in $B$ ), and $p: \mathscr{E} \rightarrow B$ by

$$
p(e, w)=w(1) \quad \text { for }(e, w) \in \mathscr{E} .
$$

Then $p: \mathscr{E} \rightarrow B$ is a Hurewicz fibration (the mapping path fibration). Note that there is a homotopy equivalence $g: E \rightarrow \mathscr{E}$ such that $p \circ g$ is homotopic to $f$.

Two Hurewicz fibrations $p_{1}: E_{1} \rightarrow B$ and $p_{2}: E_{2} \rightarrow B$ are said to be fiber homotopy equivalent if there exist maps $f: E_{1} \rightarrow E_{2}$ and $g: E_{2} \rightarrow E_{1}$ preserving fibers in the sense that $p_{2} \circ f=p_{1}$ and $p_{1} \circ g=p_{2}$ such that $g \circ f \simeq \operatorname{Id}_{E_{1}}$ and $f \circ g \simeq \operatorname{Id}_{E_{2}}$.

\section{Preliminary Results}

In this section, we review some results needed to understand the main results in §4. In particular, we give classification theorems for locally trivial bundles and Hurewicz fibrations with respect to fiber homotopy equivalence. These can be found in $[13,30]$. We begin by the following definitions.

Definition 2.1. A continuous map $p: E \rightarrow B$ onto $B$ is a quasifibration if

$$
p_{*}: \pi_{i}\left(E, p^{-1} b, e\right) \cong \pi_{i}(B, b)
$$

for all $b \in B, e \in p^{-1} b$, and $i \geq 0$.

Definition 2.2. Let $\xi=\{E, p, B, F]\}$ be a locally trivial bundle with locally compact fiber $F$. Let $\mathbf{G}$ be the space of all homotopy equivalences $F \rightarrow F$ with the compact-open topology. Define a principal G-bundle $\xi=$ $\{\widetilde{E}, \tilde{p}, B, \mathbf{G}\}$ as follows. $\widetilde{E}$ is a subspace of the space of all continuous mappings $\varphi: F \rightarrow E$ (with the compact-open topology). A map $\varphi$ is in $\widetilde{E}$ if

(1) $\varphi(F)$ is contained in some fiber $p^{-1}(x)$,

(2) $\varphi: F \rightarrow p^{-1}(x)$ is a homotopy equivalence.

The projection $\tilde{p}$ is given by $\tilde{p}(\varphi)=p(\varphi(F))$, and $\mathbf{G}$ operates in $\widetilde{E}$ by composition $\varphi(h)=\varphi \circ h$, for $\varphi \in \widetilde{E}, h \in \mathbf{G}$. 
Other essential facts about quasifibrations, principal, and universal principal quasifibrations are given in [13]. We state the following classification theorems of locally trivial bundles and Hurewicz fibrations.

Theorem 2.3 [13]. Consider locally trivial bundles $\xi=\{E, p, B, F\}$ with a fixed polyhedron $B$ as base, and locally compact fiber $F$. Let $\mathbf{H}$ be the space of homeomorphisms of $F$ with the compact-open topology, $\mathbf{G}$ be the space of homotopy equivalences of $F$ with the compact open topology, and let $\eta: \mathbf{H} \rightarrow \mathbf{G}$ be the natural inclusion. Then $\eta$ induces a homomorphism

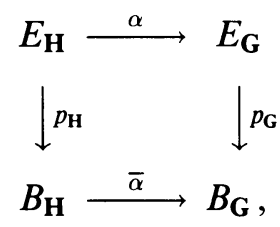

where $\left\{E_{\mathbf{H}}, p_{\mathbf{h}}, B_{\mathbf{H}}, \mathbf{H}\right\}$ is a universal principal $\mathbf{H}$-bundle and $\left\{E_{\mathbf{G}}, p_{\mathbf{G}}, B_{\mathbf{G}}, \mathbf{G}\right\}$ is a universal principal quasifibration over $\mathbf{G}$.

If we associate with every bundle $\xi$ a classifying map $\gamma: B \rightarrow B_{\mathbf{H}}$ (see [31, $\S 19])$, then

(1) two bundles $\xi, \xi^{\prime}$ are equivalent if and only if $\gamma \simeq \gamma^{\prime}$,

(2) two bundles $\xi, \xi^{\prime}$ are fiber homotopy equivalent if and only if $\bar{\alpha} \circ \gamma \simeq \bar{\alpha} \circ \gamma^{\prime}$.

Theorem 2.4 [30]. If $F$ is a finite $C W$-complex, there is a space $B_{\mathbf{G}}$ such that $\left[, B_{\mathbf{G}}\right]$ and $\mathscr{E}()$ are naturally equivalent as functors from the category of $C W$ complexes and homotopy classes of maps to the category of sets and functions, where $\mathscr{E}(X)$ denotes the set of Hurewicz fibrations $p: E \rightarrow X$ with fibers of the homotopy type of $F$.

\section{RESUltS WHEN THE BASE SPACE IS A CONTRACTIBLE MANIFOLD AND SOME EXAMPLES}

It is natural to ask whether approximate fibrations between manifolds can be approximated by locally trivial bundles. Before we consider the general case, we restrict to the case when the base space is a contractible manifold. As we mentioned in the Introduction, T. A. Chapman [6] gives criteria under which we can approximate maps into locally trivial bundles by homeomorphisms.

As an immediate consequence of Theorem 1.1, we have the following.

Corollary 3.1. Let $p: M^{m+n} \rightarrow R^{n}$ be an approximate fibration whose fiber is homotopy equivalent to a closed manifold $F$, and $m+n \geq 5$. Then for some $\pi^{-1}(\varepsilon)$-equivalence $f: M \rightarrow R^{n} \times F, \sigma(f)$ vanishes if and only if $p$ is approximated by a trivial bundle ( $\pi$ denotes the projection to $R^{n}$ ).

Proof. "Only if". It is obvious by Theorem 1.1.

"If". Suppose $p$ is approximated by a trivial bundle $q: M \rightarrow R^{n}$. Then there is a fiber preserving homeomorphism $h: M \rightarrow R^{n} \times F$. Since $p$ and $q$ are arbitrarily close, $h: M \rightarrow R^{n} \times F$ is a $\pi^{-1}(\varepsilon)$-equivalence with $\sigma(h)=0$ for arbitrary small $\varepsilon>0$.

The following corollary shows that any approximate fibration over $R^{n}$ with some special fiber is unique up to isomorphism. 
Corollary 3.2. Let $p: M^{m+n} \rightarrow R^{n}$ and $q: \widetilde{M}^{m+n} \rightarrow R^{n}$ be approximate fibrations whose fibers are homotopy equivalent to a closed manifold $F^{m}$ satisfying $S_{0}\left(T^{n} \times F\right)=0$, and $m+n \geq 5$. Then for any $\varepsilon>0$, there is a homeomorphism $h: M \rightarrow \widetilde{M}$ such that $p$ and $q \circ h$ are $\varepsilon$-close.

Proof. It is obvious by Corollary 3.1.

Sometimes surgery theory indicates that certain compact manifolds $F$ satisfy $S\left(T^{n} \times F\right)=0$. For example, it follows from [24] that if $F^{m}$ is a $K(\pi, 1)$ with $\pi$ poly $Z$ and $m+n \geq 5$, then $S\left(T^{n} \times F^{m}\right)=0$. Also any sphere $S^{m}$ satisfies $S\left(T^{n} \times S^{m}\right)=0$.

Now we consider an approximate fibration $p: M^{m+n} \rightarrow B^{n}$ between manifolds whose fiber is homotopy equivalent to a compact manifold $F$, and $B^{n}$ is contractible. Then from the homotopy exact sequence for approximate fibration, there is a homotopy equivalence $g: M \rightarrow B \times F$ such that $q \circ g$ is homotopic to $p$, where $q: B \times F \rightarrow B$ is a trivial bundle. By the homotopy lifting property of $q: B \times F \rightarrow B$, there is a homotopy equivalence $f(\simeq g)$ satisfying the commutative diagram

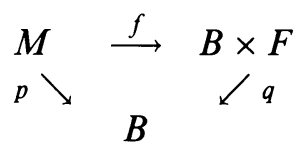

Then it easily follows that $f$ is a $q^{-1}(\varepsilon)$-equivalence for arbitrary small $\varepsilon>0$ (see Proposition 2.3 of [6]). Thus, if $F$ satisfies the hypothesis of Theorem 1.2, then $p$ can be approximated by a trivial bundle.

For example, we show that any approximate fibration over $B$ whose fiber is homotopy equivalent to $F$, a closed Riemannian manifold with nonpositive sectional curvature value, can be approximated by a trivial bundle. We will apply later the technique used to prove the above paragraph. First we begin by stating the results we will be using later in this paper. In particular, the first one is the periodicity theorem for the surgery of topological manifolds.

Theorem 3.3 [24]. For any compact topological manifold $M^{m}$ and $m \geq 5$, $S(M) \cong S\left(I^{4} \times M\right)$.

Theorem 3.4 [18]. Let $F^{m}$ be a closed Riemannian manifold whose sectional curvature value is nonpositive, and $f: M^{m} \rightarrow F^{m} \times I^{k}$ be a homotopy equivalence such that $f \mid \partial M: \partial M \rightarrow \partial\left(F \times I^{k}\right)$ is a homeomorphism, where $M$ is a compact manifold with $k+m \geq 5$. Then $f$ is homotopic to a homeomorphism via a homotopy which is constant when restricted to $\partial M$.

Proposition 3.5. Let $p: M^{m+n} \rightarrow B^{n}$ be an approximate fibration whose fiber is homotopy equivalent to $F^{m}$, which is a closed Riemannian manifold whose sectional curvature value is nonpositive, $B^{n}$ be a contractible manifold, and $m+$ $n \geq 5$. Then $p$ can be approximated by a trivial bundle.

Proof. First notice that Theorem 3.4 implies

$$
S\left(F^{m} \times I^{j}\right)=0 \text { for } m+j \geq 5 .
$$

According to Theorem 1.2, it is enough to show that

$$
S\left(F \times T^{i} \times I^{j}\right)=0 \text { for } i+j=n .
$$


We apply Theorem 3.3, and then we have

$$
S\left(F \times T^{i} \times I^{j}\right)=S\left(F \times T^{i} \times I^{j+4}\right)
$$

so that we can apply Farrell's splitting theorem [16]. We use induction on $i$. Rewrite

$$
F \times T^{i} \times I^{j+4}=F \times I^{j+4} \times T^{i-1} \times S^{1} .
$$

Suppose $[f]$ is an element of $S\left(F \times I^{j+4} \times T^{i-1} \times S^{1}\right)$, where $f: W \rightarrow F \times$ $I^{j+4} \times T^{i-1} \times S^{1}$ is a homotopy equivalence so that

$$
f \mid \partial W: \partial W \rightarrow \partial\left(F \times I^{j+4} \times T^{i-1} \times S^{1}\right)
$$

is a homeomorphism, and $W$ is a compact manifold with dimension $m+n+4$. It follows from [18] that

$$
\mathrm{Wh}\left(F \times I^{j+4} \times T^{i}\right)=0 \text { for all } i,
$$

and then we apply Farrell's splitting theorem to find a homotopy $f \simeq f_{1}(\operatorname{rel} \partial W)$ so that $f_{1}$ has the following properties;

(1) there exists a codimension 1 submanifold $N \subset W$ such that

$$
f_{1}:(W, N) \rightarrow\left(F \times I^{j+4} \times T^{i-1} \times S^{1}, F \times I^{j+4} \times T^{i-1} \times 0\right)
$$

is a homotopy equivalence of pairs, where $S^{1}=[0,1]$ with the identification $0 \sim 1$.

(2) $\left(W_{n} ; N_{0}, N_{1}\right)$ is a relative $h$-cobordism in the sense that

$$
\partial W_{n}-\operatorname{Int}\left(N_{0} \cup N_{1}\right) \cong \partial N_{0} \times I,
$$

where $W_{n}$ is the manifold obtained by cutting $W$ along $N$.

By induction on $i$, we have $S\left(F \times I^{j+4} \times T^{i-1}\right)=0$. Thus, we may assume that $f_{1}: W_{n} \rightarrow F \times I^{j+4} \times T^{i-1} \times[0,1]$ is a homotopy equivalence so that $f_{1} \mid N_{i} \quad(i=$ $0,1)$ is a homeomorphism, and then $f_{1}$ is homotopic to a homeomorphism $(\operatorname{rel} \partial)$. This implies the given map $f_{1}: W \rightarrow F \times I^{j+4} \times T^{i}$ is homotopic to a homeomorphism $(\operatorname{rel} \partial W)$. In other words,

$$
S\left(F \times I^{j+4} \times T^{i}\right)=S\left(F \times I^{j} \times T^{i}\right)=0 \text { for } i+j=n .
$$

Hence the conclusion follows from Theorem 1.2.

Next we consider examples of approximate fibrations which cannot be approximated by locally trivial bundle maps. As we mentioned in the Introduction, L. S. Husch has described an approximate fibration $p: M^{n} \rightarrow S^{1}(n \geq 6)$ such that $p$ cannot be approximated by a locally trivial bundle map [23]. Before we proceed further, we give a result [6], which is used in Examples 3.7 and 3.8.

Proposition 3.6. Let $B$ be a space which is locally polyhedral, $\alpha$ an open cover of $B$, and $m \geq 5$ be an integer. Then there exists an open cover $\beta$ of $B$ so that if $M^{m}$ is a manifold $(\partial M=\varnothing)$ and $f: M \rightarrow B$ is a $\beta$-fibration, then $f$ is $\alpha$-close to an approximate fibration $p: M \rightarrow B$.

In the following examples, we show that some approximate fibrations $p: M^{m+n}$ $\rightarrow T^{n}$ and $p: M^{m+n} \rightarrow R^{n}$ whose fibers are homotopy equivalent to $N^{m}=$ $L^{3} \times T^{m-3}$ cannot be approximated by locally trivial bundle maps for $m+n \geq 6$, where $L^{3}$ is a Lens space with $\pi_{1}(L) \cong Z_{p^{2}} \quad(p$ : prime $)$ and $T^{n}$ is the $n$ dimensional torus. 
Example 3.7. Let $L^{3}$ be a 3-dimensional Lens space with $\pi_{1}(L) \cong Z_{p^{2}} \quad(p$ : prime), and $T^{m-2}$ be the $(m-2)$-dimensional torus. We consider $M_{0}^{m+1}=$ $L^{3} \times T^{m-2}(m+1 \geq 6)$. Rewrite $M_{0}=L^{3} \times T^{m-2}$ as $N^{m} \times S^{1}$, where $N=L^{3} \times T^{m-3}$.

Farrell and Hsiang [16] showed that there exists an $h$-cobordism $\left(W^{n+2}\right.$; $M_{0}^{m+1}, M_{1}^{m+1}$ ) such that $M_{0}$ is not homeomorphic to $M_{1}$, but the obstruction to splitting $M_{1}$ into $N^{\prime} \times R$ vanishes, where $N^{\prime}$ is a codimension 1 submanifold of $M_{1}$. We consider a homotopy equivalence $f_{1}: M_{1} \rightarrow M_{0}$, which is the composition of the following maps

$$
M_{1} \stackrel{\text { inclusion }}{\longrightarrow} W \underset{\text { retract }}{\stackrel{\text { deformation }}{\longrightarrow}} M_{0} \text {. }
$$

Let $\pi: M_{0} \rightarrow S^{1}$ be the projection onto the second factor. We consider the following pull-back diagrams

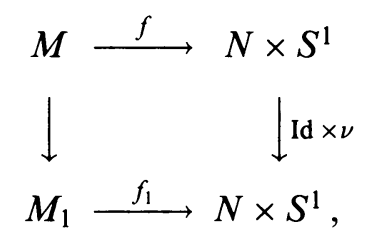

and

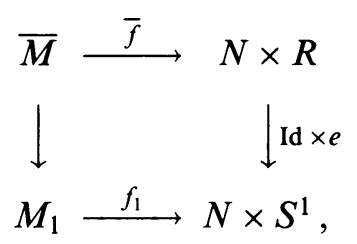

where $\nu: S^{1} \rightarrow S^{1}$ is defined by $\nu(z)=z^{k} \quad\left(k:\right.$ positive integer) and $e: S^{1} \rightarrow$ $S^{1}$ by $e(x)=e^{\pi i x}$.

Since the obstruction to splitting $M_{1}$ into $N^{\prime} \times R$ vanishes, we can restore $f_{1}$ by the wrapping up process (see $[6,28]$ ), and hence there exists a homeomorphism $h: M \rightarrow M_{1}$ such that $f \circ h \simeq f_{1}$ (see Lemma 7.3, [6]).

If $k$ is large enough, $\pi \circ f: M \rightarrow S^{1}$ is close to an approximate fibration $p: M \rightarrow S^{1}$ by Proposition 3.6, where $\pi: N \times S^{1} \rightarrow S^{1}$ is the projection.

Claim. $p: M \rightarrow S^{1}$ cannot be approximated by a locally trivial bundle.

Otherwise, $p$ is approximated by a locally trivial bundle $q: M \rightarrow S^{1}$. Then $q$ is $\varepsilon$-homotopic to $\pi \circ f$ for arbitrary small $\varepsilon>0$. By the homotopy lifting property, there exists a fiber homotopy equivalence $g: M \rightarrow N \times S^{1}$ such that $f \simeq g$.

Since $\chi\left(S^{1}\right)=0$, the Whitehead torsion $\tau(g)$ in $\mathrm{Wh}\left(N \times S^{1}\right)$ is zero [2], and hence $\tau\left(f_{1}\right)=\tau(f)=0$. Then the $h$-cobordism $\left(W ; M_{0}, M_{1}\right)$ is homeomorphic to the product. Thus, $M_{0} \cong M_{1}$. This is a contradiction. Now consider $M_{0}=L^{3} \times T^{m-2}=N \times S^{1} \times S^{1}$, where $N=L^{3} \times T^{m-4}$. Form the following pull-back

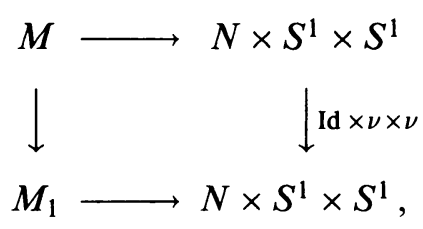


where $\nu: S^{1} \rightarrow S^{1}$ by $\nu(z)=z^{k}$ for integer $k>0$. But the above pull-back factors into the two pull-backs,

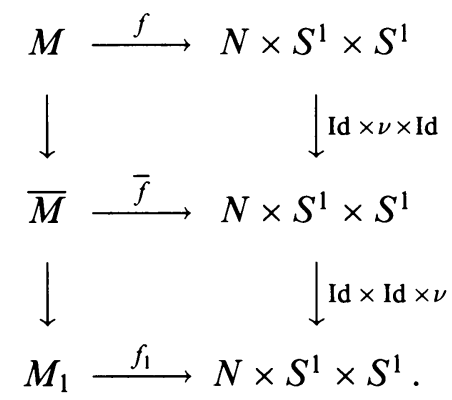

By the same argument, we conclude that there is a homeomorphism $h: M \rightarrow M_{1}$ such that $f_{1} \circ h \simeq f$. Hence, for large enough $k, \pi \circ f: M \rightarrow S^{1} \times S^{1}$ is close to an approximate fibration $p: M \rightarrow S^{1} \times S^{1}$, But $p$ cannot be approximated by a locally trivial bundle, where $\pi: N \times S^{1} \times S^{1} \rightarrow S^{1} \times S^{1}$ is the projection.

Similarly we can extend so that there are some approximate fibrations $p: M^{m+n} \rightarrow T^{n} \quad(m+n \geq 6)$ whose fibers are homotopy equivalent to $N^{m}=$ $L^{3} \times T^{m-3}$ which cannot be approximated by locally trivial bundle maps.

Example 3.8. In the previous example, there exists an $h$-cobordism $\left(W^{m+2}\right.$; $M_{0}^{m+1}, M_{1}^{m+1}$ ) such that $M_{0}$ is not homeomorphic to $M_{1}$, but the obstruction to splitting $M_{1}$ into $N^{\prime} \times R$ vanishes for $M_{0}=L^{3} \times T^{m-2}$, where $N^{\prime}$ is a codimension 1 submanifold of $M_{1}$. Without loss of generality we assume a natural homotopy equivalence $f_{1}: M_{1} \rightarrow M_{0}=N \times S^{1}$ as a $\pi^{-1}(\varepsilon)$-equivalence for small enough $\varepsilon>0$.

Consider the pull-back diagram

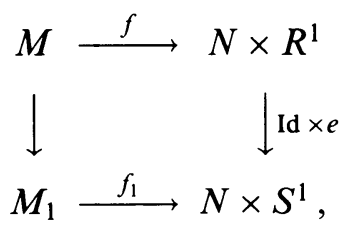

where $e: R^{1} \rightarrow S^{1}$ is the covering projection defined by $e(x)=e^{\pi i x}$ for $x \in$ $R^{1}$. Then $f$ is a $q^{-1}(\delta)$-equivalence, $\delta$ depends on the size of $\varepsilon$, and $q: N \times$ $R^{1} \rightarrow R^{1}$ is the projection onto the 2 nd factor.

For such an $f, q \circ f: M \rightarrow R^{1}$ is a $2 \delta$-fibration, and hence $q \circ f$ is close to an approximate fibration $p: M \rightarrow R^{1}$ by Proposition 3.6.

Claim. $p: M \rightarrow R^{1}$ cannot be approximated by a trivial bundle.

Otherwise, there exists a homeomorphism $h: M \rightarrow N \times R^{1}$ such that $h$ is $\pi^{-1}(\varepsilon)$-close to $f$. Since the obstruction to splitting $M_{1}$ is zero, by the wrapping up process, we can recover $f_{1}: M \rightarrow N \times S^{1}$. In other words, there is a $q^{-1}(\varepsilon)$-equivalence $\hat{f}: M_{1} \rightarrow N \times S^{1}$ such that $\hat{f} \simeq f_{1}$. By the uniqueness of the wrapping up process [6], we find a homeomorphism $\hat{h}: M_{1} \rightarrow N \times S^{1}=M_{0}$. This is a contradiction.

By the similar inductive procedure in Example 3.7, we can extend so that there are some approximate fibrations $p: M^{m+n} \rightarrow R^{n}(m+n \geq 6)$ whose fiber is homotopy equivalent to $N^{m}=L^{3} \times T^{m-3}$ which cannot be approximated by trivial bundle maps. 


\section{CONDITIONS ON APPROXIMATING APPROXIMATE FIBRATIONS BY LOCALLY TRIVIAL BUNDLE MAPS}

In this section, we find some conditions so that approximate fibrations between manifolds can be approximated by locally trivial bundle maps.

Recall that for a closed manifold $F, \mathbf{H}$ is the space of self-homeomorphisms of $F$ with the compact-open topology, and $\mathbf{G}$ is the space of self-homotopy equivalences of $F$ with the compact-open topology. We define the following group;

$$
K_{k}=\operatorname{ker}\left\{\pi_{k}(\mathbf{H}, e) \rightarrow \pi_{k}(\mathbf{G}, e)\right\}
$$

for all $k \geq 0$, where $e$ is the identity map of $F$.

Lemma 4.1. Let $F$ be a closed manifold, $q: E \rightarrow \dot{\Delta}^{k}(k \geq 2)$ be a locally trivial bundle with fiber $F$. Suppose $q$ is fiber homotopy equivalent to the product bundle $\pi: \dot{\Delta}^{k} \times F \rightarrow \dot{\Delta}^{k}$. Then there is an obstruction $\sigma(q)$ in $K_{k-2}$ which vanishes if and only if $q$ extends to a locally trivial bundle $\tilde{q}: \widetilde{E} \rightarrow \Delta^{k}$ with fiber $F$.

Proof. By Theorem 2.3 (classification theorem), for the equivalence class of such a locally trivial bundle $q: E \rightarrow \dot{\Delta}^{k} \quad(k \geq 2)$, there is the associated element in $\operatorname{ker}\left\{\pi_{k-1}\left(B_{\mathbf{H}}\right) \rightarrow \pi_{k-1}\left(B_{\mathbf{G}}\right)\right\}$. Notice that we can identify $\operatorname{ker}\left\{\pi_{k-1}\left(B_{\mathbf{H}}\right) \rightarrow \pi_{k-1}\left(B_{\mathbf{G}}\right)\right\}$ with $K_{k-2}$. In fact, from the commutative diagram,

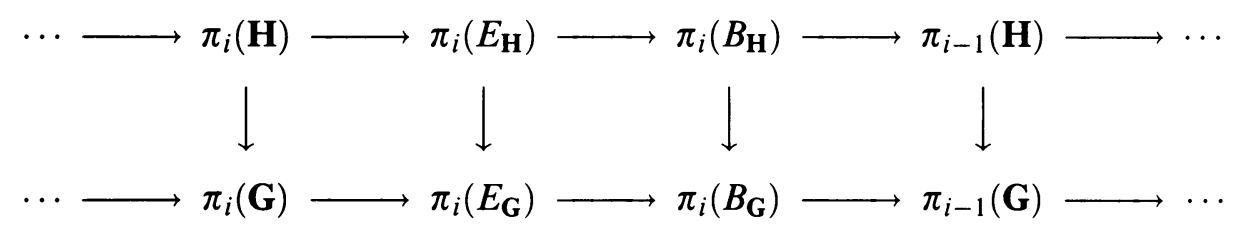

and $\pi_{i}\left(E_{\mathbf{H}}\right)=\pi_{i}\left(E_{\mathbf{G}}\right)=0$, we have

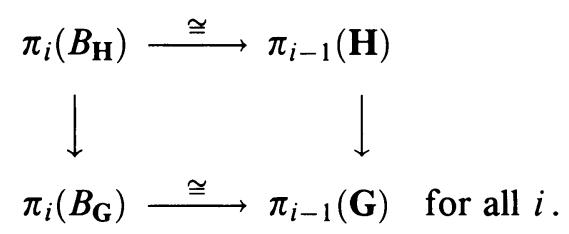

Hence, we can define $\sigma(q)$ as the corresponding element in $K_{k-2}$. It is obvious that $\sigma(q)$ is well defined for the equivalence class of $q: E \rightarrow \dot{\Delta}^{k}$ by the classification theorem.

Suppose the obstruction $\sigma(q)$ vanishes. Then $q: E \rightarrow \dot{\Delta}^{k}$ is a trivial bundle, and hence there is an isomorphism $\alpha: \dot{\Delta}^{k} \times F \rightarrow E$. If we define $\widetilde{E}$ by

$$
\widetilde{E}=\Delta^{k} \times F \cup_{\alpha} E,
$$

then $q: E \rightarrow \dot{\Delta}^{k}$ extends to a locally trivial bundle $\tilde{q}: \widetilde{E} \rightarrow \Delta^{k}$.

It is obvious that $\sigma(q)$ vanishes if $q: E \rightarrow \dot{\Delta}^{k}$ has extension $\tilde{q}: \widetilde{E} \rightarrow \Delta^{k}$.

Lemma 4.2. Let $p: \mathscr{E} \rightarrow B$ be a Hurewicz fibration whose fiber is homotopy equivalent to a closed manifold $F$, and $B$ be a polyhedron such that $B=$ $B^{(k)} \cup \Delta^{k+1}$, where $B^{(k)}$ is $k$-skeleton and $\Delta^{k+1}$ is a single $k$-simplex. Suppose 
$p|: \mathscr{E}| \rightarrow B^{(k)}$ is fiber homotopy equivalent to a locally trivial bundle $q: E \rightarrow B^{(k)}$ with fiber $F$. Then there is an obstruction $\sigma\left(q \mid \dot{\Delta}^{k+1}\right)$ in $K_{k-1}$ which vanishes if and only if there is a locally trivial bundle $\tilde{q}: \widetilde{E} \rightarrow B$, an extension of $q$, which is fiber homotopy equivalent to $p: \mathscr{E} \rightarrow B$.

Proof. "Only if". Assume that $\sigma\left(q \mid \dot{\Delta}^{k+1}\right)$ in $K_{k-1}$ vanishes. By Lemma 4.1, $q: E \rightarrow B^{(k)}$ extends to a locally trivial bundle $\tilde{q}: \widetilde{E} \rightarrow B$. Now we apply Theorem 2.4 (the classification theorem of Hurewicz fibrations). For Hurewicz fibrations $p: \mathscr{E} \rightarrow B$ and $\tilde{q}: \widetilde{E} \rightarrow B$, there are the associated maps $\gamma_{1}, \gamma_{2}: B \rightarrow$ $B_{\mathbf{G}}$, respectively. Again by Theorem $2.4, \gamma_{1} \mid B^{(k)}$ and $\gamma_{2} \mid B^{(k)}$ are homotopic. It is easy to get a homotopy between $\gamma_{1}$ and $\gamma_{2}$ by collapsibility of $\Delta^{k+1}$, and hence $p: \mathscr{E} \rightarrow B$ is fiber homotopy equivalent to $\tilde{q}: \widetilde{E} \rightarrow B$.

"If". It is obvious.

Remark. For a 1-dimensional polyhedron $B$ and a Hurewicz fibration $p: \mathscr{E} \rightarrow$ $B$ whose fiber is homotopy equivalent to $F$, there exists a locally trivial bundle $q: E \rightarrow B$ with fiber $F$ which is fiber homotopy equivalent to $p: \mathscr{E} \rightarrow B$, since every locally trivial bundle over the 0 -skeleton extends to a locally trivial bundle over the 1-skeleton.

We consider a Hurewicz fibration $p: \mathscr{E} \rightarrow B^{2}$ (polyhedron) whose fiber is homotopy equivalent to a closed manifold $F$. By the above remark, there exists a locally trivial bundle $q: E \rightarrow B^{(1)}$ with fiber $F$, which is fiber homotopy equivalent to $p|: \mathscr{E}| \rightarrow B^{(1)}$. Then we have the following result.

Theorem 4.3. Let $p: \mathscr{E} \rightarrow B^{2}$ (polyhedron) be a Hurewicz fibration whose fiber is homotopy equivalent to closed manifold $F$. Then for some locally trivial bundle $q: E \rightarrow B^{(1)}$ with fiber $F$, which is fiber homotopy equivalent to $p|: \mathscr{E}| B^{(1)} \rightarrow$ $B^{(1)}$, there is an obstruction in the direct product of copies of $K_{0}$ which vanishes if and only if $p: \mathscr{E} \rightarrow B$ is fiber homotopy equivalent to a locally trivial bundle $\tilde{q}: \widetilde{E} \rightarrow B$ with fiber $F$.

Proof. For each $\dot{\Delta}^{2}$ in $B^{(1)}$, there exists an obstruction $\sigma\left(q \mid \dot{\Delta}^{2}\right)$ in $K_{0}$ which vanishes if and only if $q$ extends to a locally trivial bundle over $B^{(1)} \cup \Delta^{2}$. Thus, in order to extend $q: E \rightarrow B^{(1)}$ to a locally trivial bundle over $B$, we have an obstruction as follows:

$$
\tau(q)=\left(\sigma\left(q \mid \dot{\Delta}_{i}^{2}\right)\right) \in \prod_{i \in \mathbf{A}} K_{0}^{(i)},
$$

where $\mathbf{A}$ is the collection of indices of $\dot{\Delta}^{2}$ in $B^{(1)}$ and $K_{0}^{i}$ is a copy of $K_{0}$ for each $i \in \mathbf{A}$. Then $\tau(q)$ is well defined for the equivalence class of $q: E \rightarrow B^{(1)}$ since $\sigma\left(q \mid \dot{\Delta}_{i}^{2}\right)$ is well defined for each $\dot{\Delta}_{i}^{2}$ in $B^{(1)}$. Therefore, the proof follows easily from Lemma 4.2 .

For a $k$-dimensional polyhedron $B^{k} \quad(k \geq 2)$ and a Hurewicz fibration $p: \mathscr{E} \rightarrow B$, we inductively define obstructions in the direct product of copies of $K_{i}$ for $0 \leq i \leq k-2$ so that if all obstructions vanish, then $p: \mathscr{E} \rightarrow B$ is fiber homotopy equivalent to a locally trivial bundle $q: E \rightarrow B$.

Theorem 4.4. Let $p: \mathscr{E} \rightarrow B$ be a Hurewicz fibration whose fiber is homotopy equivalent to a closed manifold $F$, where $B$ is a $k$-dimensional polyhedron. Then for some locally trivial bundle $q: E \rightarrow B^{(1)}$ with fiber $F$, which is fiber 
homotopy equivalent to $p|: \mathscr{E}| \rightarrow B^{(1)}$, there are obstructions, with increasing order over skeleta, in the direct products of copies of $K_{i}$ for $0 \leq i \leq k-2$ which vanish if and only if $p: \mathscr{E} \rightarrow B$ is fiber homotopy equivalent to a locally trivial bundle $\tilde{q}: \widetilde{E} \rightarrow B$ with fiber $F$.

Now we consider an approximate fibration $p: M \rightarrow B$ whose fiber is homotopy equivalent to a closed manifold $F$. First we observe the following.

Lemma 4.5. Let $p: M \rightarrow B$ be an approximate fibration, and $q: E \rightarrow B$ be a Hurewicz fibration. Suppose $g: M \rightarrow E$ is a homotopy equivalence so that $p$ is homotopic to $q \circ g$. Then $p^{-1}(b)$ is shape equivalent to $q^{-1}(b)$ for each $b \in B$. Proof. By the homotopy lifting property for $q$, we find a homotopy equivalence $f(\simeq g)$ such that $p=q \circ f$.

Consider the following long exact sequence

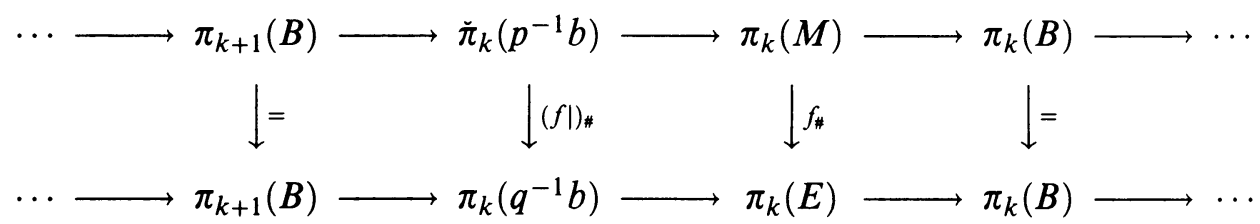

where $\check{\pi}_{k}\left(p^{-1} b\right)$ denotes the $k$ th shape homotopy group of $p^{-1}(b)$ (analogous to the homotopy group). From the five lemmas, $f \mid p^{-1} b$ induces isomorphisms of $\check{\pi}_{k}\left(p^{-1} b\right)$ and $\pi_{k}\left(q^{-1} b\right)$ for all $k$. Notice that $p^{-1} b$ is FANR [8]. The Whitehead theorem (for shape) implies $f \mid p^{-1} b: p^{-1} b \rightarrow q^{-1} b$ is a shape equivalence for all $b \in B$ [26].

For an approximate fibration $p: M \rightarrow B$ whose fiber is homotopy equivalent to a closed manifold $F$, we consider the mapping path fibration $\tilde{p}: \mathscr{E} \rightarrow B$, where

$$
\mathscr{E}=\left\{(e, w) \in M \times B^{I} \mid p(e)=w(0)\right\} .
$$

$\left(B^{I}\right.$ is the space of paths in $B$ ), and $\tilde{p}: \mathscr{E} \rightarrow B$ is defined by

$$
\tilde{p}(e, w)=w(1) \quad \text { for }(e, w) \in \mathscr{E} .
$$

By Lemma $4.5, p^{-1}(b)$ is shape equivalent to $\tilde{p}^{-1}(b)$ for each $b \in B$. Since $\mathscr{E}$ and all fibers of $\tilde{p}$ are ANR's [1], all fibers of $\tilde{p}$ are homotopy equivalent to a closed manifold $F$. This observation gives us the following.

Proposition 4.6. Let $p: M \rightarrow B$ be an approximate fibration whose fiber is homotopy equivalent to a closed manifold $F$. Then there exists a locally trivial bundle $q: E \rightarrow B$ with fiber $F$ and $a q^{-1}(\varepsilon)$-equivalence $f: M \rightarrow E$ for arbitrary small $\varepsilon>0$ if and only if the mapping path fibration $\tilde{p}: \mathscr{E} \rightarrow B$ of $p$ is fiber homotopy equivalent to $q: E \rightarrow B$.

Proof. "Only if". Suppose $f_{1}: M \rightarrow \mathscr{E}$ is a $\tilde{p}^{-1}\left(\varepsilon_{1}\right)$-equivalence for small enough $\varepsilon_{1}>0$, and $f: M \rightarrow E$ is a $q^{-1}(\varepsilon)$-equivalence for small enough $\varepsilon>0$. If $\tilde{f}: E \rightarrow M$ is a homotopy inverse of $f$, then $\tilde{p} \circ f_{1} \circ \tilde{f}$ is homotopic to $q: E \rightarrow B$. By the homotopy lifting property for Hurewicz fibration, there is a fiber homotopy equivalence $q: E \rightarrow \mathscr{E}$.

"If". Suppose $g: \mathscr{E} \rightarrow E$ is a fiber homotopy equivalence. Since there exists a $\tilde{p}^{-1}(\varepsilon)$-equivalence $f_{1}: M \rightarrow \mathscr{E}$, the composition $g \circ f_{1}$ is a $q^{-1}(\varepsilon)$ equivalence for small enough $\varepsilon>0$.

As a result, we have the following theorems. 
Theorem 4.7. Let $F^{m}(m \geq 3)$ be a closed manifold satisfying $S\left(F \times I^{i} \times T^{j}\right)=$ 0 for $i+j=2$, and $p: M^{m+2} \rightarrow B^{2}$ be an approximate fibration whose fiber is homotopy equivalent to $F^{m}$, where $M$ is a manifold $(\partial M=\varnothing)$ and $B$ is a polyhedron. Then for some locally trivial bundle $q: E \rightarrow B^{(1)}$, there is an obstruction in the direct product of copies $K_{0}$ which vanish if and only if $p$ is approximated by a locally trivial bundle with fiber $F$.

Proof. It follows from Theorem 1.2, Theorem 4.3, and Proposition 4.6.

Remark. As before, for a 1-dimensional polyhedron $B$ and a closed manifold $F^{m} \quad(m \geq 4)$ satisfying $S\left(F \times I^{i} \times T^{j}\right)=0$ for $i+j=1$, any approximate fibration $p: M^{m+1} \rightarrow B^{1}$ with fiber $F$ can be approximated by a locally trivial bundle.

For a closed manifold $F$, let $C(F)$ denote the space of all concordances of $F$, i.e. $C(F)$ is the function space of all homeomorphism of $F \times[0,1]$ onto itself that fix $F \times 0$. We have the following corollary.

Corollary 4.8. Let $p: M^{m+2} \rightarrow B^{2}$ be an approximate fibration between manifolds $(\partial M=\varnothing)$ such that each fiber is homotopy equivalent to a closed manifold $F^{m}$ with the property $\pi_{0}(C(F))=0$ and $S(F \times I)=0$. Then there exists a locally trivial bundle $q: E^{m+2} \rightarrow B^{2}$ with fiber $F$, and a $q^{-1}(\varepsilon)$-equivalence $f: M \rightarrow E$ for small enough $\varepsilon>0$.

Proof. $\pi_{0}(C(F))=0$ and $S(F \times I)=0$ imply that the inclusion $\mathbf{H} \rightarrow \mathbf{G}$ induces a $\pi_{0}$-monomorphism. Thus, by Theorem 4.7 , the conclusion follows.

Remark. For simply connected closed manifold $F^{m}(m \geq 4)$, it is well known that $\pi_{0}(C(F))=0$;

(1) for $n \geq 5$, it is due to J. Cerf [3],

(2) for $n=4$, it is due to F. Quinn [27].

If we combine Theorem 1.2, Theorem 4.4 and Proposition 4.6, we get the similar result for the codimension $k(\geq 2)$ case.

Theorem 4.9. Let $F^{m}$ be a closed manifold satisfying $S\left(F \times I^{i} \times T^{j}\right)=0$ for $i+j=n$, and $p: M^{m+n} \rightarrow B^{n}(m+n \geq 5)$ be an approximate fibration whose fiber is homotopy equivalent to $F^{m}$, where $M$ is a manifold $(\partial M=\varnothing)$ and $B^{n}$ is a polyhedron. Then for some locally trivial bundle $q: E \rightarrow B^{(1)}$ with fiber $F$, there are obstructions, with increasing order over skeleta, in the direct products of copies of $K_{i}$ for $0 \leq i \leq k-2$, which vanish if and only if $p$ is approximated by a locally trivial bundle with fiber $F$.

The following will be the essential key to prove Theorem 1.3.

Theorem 4.10. Let $p: M^{m+n} \rightarrow B^{n}$ be an approximate fibration whose fiber is homotopy equivalent to a closed manifold $F^{m}$, where $M$ is a manifold $(\partial M=$ $\varnothing)$ and $B$ is an n-dimensional polyhedron. Suppose the natural inclusion map $\mathbf{H} \rightarrow \mathbf{G}$ induces $\pi_{i}$-monomorphisms for $0 \leq i \leq n-2$. Then there exists a locally trivial bundle map $q: E^{m+n} \rightarrow B^{n}$ with fiber $F^{m}$ and a $q^{-1}(\varepsilon)$-equivalence $f: M \rightarrow E$ for small enough $\varepsilon>0$.

Proof. Since $\pi_{i}(\mathbf{H}, e) \rightarrow \pi_{i}(\mathbf{G}, e)$ is a monomorphism for $0 \leq i \leq n-2$, all $K_{i}$ 's are zero. Recall that

$$
K_{i}=\operatorname{ker}\left\{\pi_{i}(\mathbf{H}, e) \rightarrow \pi_{i}(\mathbf{G}, e)\right\} .
$$


By Proposition 4.6, we can replace $p: M \rightarrow B$ by the mapping path fibration $\tilde{p}: \mathscr{E} \rightarrow B$. Since $K_{i}=0$ for $0 \leq i \leq n-2$, all obstructions vanish in Theorem 4.4 , and hence $\tilde{p}: \mathscr{E} \rightarrow B$ is fiber homotopy equivalent to a locally trivial bundle $q: E \rightarrow B$. The conclusion follows again by Proposition 4.6.

Finally, if we combine Theorems 1.2 and 4.7, we obtain Theorem 1.3 giving the condition whether approximate fibrations can be approximated by a locally trivial bundle maps or not.

\section{Applications}

In this section, we give some specific examples of closed manifolds which are homotopy equivalent to fibers of approximate fibrations which can always be approximated by locally trivial bundles.

First, we consider the codimension 1 case. Notice that if $F$ is any closed $n$-manifold and $p: M^{m+1} \rightarrow S^{1}$ is a proper map such that each $p^{-1}(b)$ is locally flat in $M^{m+1}$ and homotopy equivalent to $F$, then $p: M^{m+1} \rightarrow S^{1}$ is an approximate fibration [9]. Thus, we have the following result.

Theorem 5.1. Given a proper map $p: M^{m+1} \rightarrow S^{1}(m \geq 4)$ such that each $p^{-1}(b)$ is locally flat in $M^{m+1}$ and homotopy equivalent to a closed manifold $F^{m}, p$ can be approximated by a locally trivial bundle provided $S\left(F \times I^{j} \times T^{j}\right)=$ 0 for $i+j=1$.

Proof. Since $p: M^{m+1} \rightarrow S^{1}$ is an approximate fibration, the conclusion follows from the remark below Theorem 4.7.

Corollary 5.2. If $F^{m} \quad(m \geq 4)$ is a closed Riemannian manifold with nonpositive sectional curvature value in the above theorem, then a proper map $p: M^{m+1} \rightarrow S^{1}$ can be approximated by a locally trivial bundle map.

Proof. Theorem 3.4 indicates $S\left(F^{m} \times I^{j}\right)=0$ for $m+j \geq 5$. From the proof of Proposition 3.5, we have $S\left(F \times T^{i} \times I^{j}\right)=0$ for $i+j=1$. By Theorem 5.1 , we obtain the result.

Remark. If $F^{m}(m \geq 4)$ is either a closed, aspherical manifold with poly $Z$-cyclic fundamental group or a sphere, we have the same result.

Next, we consider the codimension 2 case. In [10], R. J. Daverman showed that every closed orientable surface $F^{2}$ except $\chi\left(F^{2}\right)=0$ is a codimension 2 fibrator. In other words, for an arbitrary manifold $M^{4}$ and any usc decomposition $G$ of $M$ into copies of $F^{2}$, the decomposition map $p: M^{4} \rightarrow B^{2}$ $(=M / G)$ is an approximate fibration. Then we show that $M^{4} \times S^{1}$ has a locally trivial bundle structure. We begin with the following results to show that the induced homomorphism $\pi_{0}(\mathbf{H}, e) \rightarrow \pi_{0}(\mathbf{G}, e)$ is an isomorphism.

Theorem 5.3 [32]. Let $F^{3}$ be a closed Haken 3-manifold. Then the induced homomorphism $\pi_{0}(\mathbf{H}, e) \rightarrow \pi_{0}(\mathbf{G}, e)$ is an isomorphism.

Theorem 5.4 [25]. Suppose $h: S^{1} \times S^{2} \rightarrow S^{1} \times S^{2}$ is a homeomorphism homotopic to the identity. Then $h$ is isotopic to the identity.

As a consequence, we have

Theorem 5.5. Let $F^{2}$ be a closed orientable surface except $\chi\left(F^{2}\right)=0$. Then, for the decomposition map $p: M^{4} \rightarrow B^{2}$, the composition map $\pi \circ(p \times \mathrm{Id}): M^{4} \times$ 
$S^{1} \rightarrow B^{2}$ can be approximated by a locally trivial bundle, where $\pi$ is the projection from $B^{2} \times S^{1}$ to $B^{2}$.

Proof. For a usc decomposition $G$ of $M^{4}$ into copies of $F^{2}$, we denote a usc decomposition $G^{\prime}$ of $M^{4} \times S^{1}$ by $G^{\prime}=\left\{g \times S^{1} \mid g \in G\right\}$. Then $\pi \circ$ $(p \times$ Id $): M^{4} \times S^{1} \rightarrow B^{2}$ is the decomposition map. If $\chi(F)<0$, then it easily follows that $F^{2} \times S^{1}$ is a Haken 3-manifold. Thus, Theorem 5.3 and Theorem 5.4 imply that $\pi_{0}(\mathbf{H}, e) \rightarrow \pi_{0}(\mathbf{G}, e)$ is an isomorphism. So it suffices to show that

$$
S\left(F \times S^{1} \times T^{i} \times I^{j}\right)=0 \text { for } i+j=2 .
$$

However, it is well known that

$$
S\left(F^{2} \times I^{j}\right)=0 \text { for } 2+j \geq 5[6,24],
$$

and

$$
\mathrm{Wh}\left(F \times T^{i}\right)=0 \text { for all } i[18] .
$$

By the same argument as Proposition 4.6, we can show that

$$
S\left(F \times S^{1} \times T^{i} \times I^{j}\right)=0 \text { for } i+j=2 .
$$

Then the conclusion follows by Theorem 1.3.

Similarly, for a sphere $S^{n}$, we know that the induced homomorphism $\pi_{0}(\mathbf{H}, e) \rightarrow \pi_{0}(\mathbf{G}, e)$ is an isomorphism. Also, $S^{n}$ is a codimension $2 \mathrm{fi}-$ brator for $n \geq 2$ [12]. Therefore, the following is an easy consequence of this observation.

Theorem 5.6. Let $G$ be a usc decomposition of arbitrary manifold $M^{m+2}$ into copies of $S^{n}(n \geq 3)$. Then the decomposition map $p: M^{m+2} \rightarrow B^{2}$ can be approximated by a locally trivial bundle map.

Proof. As before, we can easily show that

$$
S\left(S^{n} \times T^{i} \times I^{j}\right)=0 \text { for } i+j=2[6] .
$$

Finally, we show that if $F^{2}$ is a closed orientable surface with $\chi(F) \leq 0$, then every approximate fibration $p: M^{m+2} \rightarrow B^{n} \quad(n \geq 3)$ whose fiber is homotopy equivalent to $F^{2}$ can be approximated by a locally trivial bundle, which is the promised generalization of R. E. Goad's result [19].

Lemma 5.7. Let $F^{2}$ be a closed orientable surface with $\chi(F) \leq 0$. Then the natural inclusion $\mathbf{H} \rightarrow \mathbf{G}$ induces $\pi_{i}$-isomorphisms for all $i \geq 0$.

Proof. If $F^{2}$ is the torus, by work of M. E. Hamstrom [21], the inclusion $\mathbf{H} \rightarrow \mathbf{G}$ is a homotopy equivalence. We assume that $F^{2}$ has genus at least 2. For $i=0, \pi_{0}(\mathbf{H}, e) \rightarrow \pi_{0}(\mathbf{G}, e)$ is an isomorphism (for more details, see [14]). M. E. Hamstrom has shown $\pi_{i}(\mathbf{H}, e)=0$ for all $i \geq 1$ [22]. Since $F^{2}$ is aspherical, we have

$$
\pi_{i}(\mathbf{G}, e)=0 \text { for } i>1
$$

and

$$
\pi_{1}(\mathbf{G}, e)=Z\left(\pi_{1}(F)\right), \text { the center of } \pi_{1}(F) \quad(\text { see [20]) } .
$$

Thus, we have

$$
\pi_{i}(\mathbf{G}, e)=0 \text { for all } i \geq 1
$$


since $Z\left(\pi_{1}(F)\right)=0$ for $\chi(F)<0$. Therefore, the induced homomorphism $\pi_{i}(\mathbf{H}, e) \rightarrow \pi_{i}(\mathbf{G}, e)$ are isomorphisms for all $i \geq 0$.

Theorem 5.8. For an approximate fibration $p: M^{n+2} \rightarrow B^{n}$ between manifolds whose fiber is homotopy equivalent to an orientable surface $F^{2}$ with $\chi(F) \leq 0$ and $n \geq 3, p: M^{n+2} \rightarrow B^{n}$ can be approximated by a locally trivial bundle.

Proof. By Lemma 5.7, the induced homomorphism $\pi_{i}(\mathbf{H}, e) \rightarrow \pi_{i}(\mathbf{G}, e)$ are isomorphisms for all $i \geq 0$. We consider two cases.

Case 1. $B$ is a polyhedron.

As before, it suffices to show that

$$
S\left(F \times T^{i} \times I^{j}\right)=0 \text { for } i+j=n .
$$

But this is obtained from the proof of Proposition 3.5 because $F$ is a closed Riemannian manifold whose sectional curvature value is nonpositive.

Case 2. $B$ is not a polyhedron.

We can choose a polyhedron $B_{1}$ which is homotopy equivalent to $B$ [3]. We denote a homotopy equivalence by $f: B_{1} \rightarrow B$. If we replace $p: M \rightarrow B$ by the mapping path fibration $\tilde{p}: \mathscr{E} \rightarrow B$, then we have the following diagram

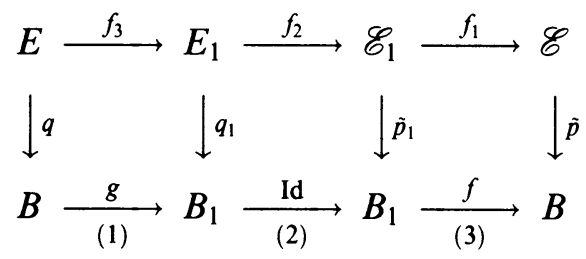

Here $g$ is a homotopy inverse of $f,(1)$ and (3) are pull-back diagrams which are obtained by the same argument as Case 1. Thus, $q: E_{1} \rightarrow B_{1}$ is a locally trivial bundle with fiber $F, f_{2}$ is a fiber homotopy equivalence, and $q: E \rightarrow B$ is also a locally trivial bundle. If we define $\tilde{f}=f_{1} \circ f_{2} \circ f_{3}$, then $\tilde{f}$ is a homotopy equivalence and $\tilde{p} \circ \tilde{f}$ is homotopic to $q$. By the homotopy lifting property, $\tilde{p}: \mathscr{E} \rightarrow B$ is fiber homotopy equivalent to a locally trivial bundle $q: E \rightarrow B$. Hence, the conclusion follows by Theorem 1.2 and Proposition 4.6.

R. J. Daverman showed [11] that every closed orientable surface $F^{2}$ of genus at least 2 is a PL-fibrator (= PL codimension $k$-fibrator for all $k$ ). As a consequence of Theorem 5.8, we have the following.

Corollary 5.9. If $B$ is a polyhedron and $p: M^{n+2} \rightarrow B^{n} \quad(n \geq 3)$ is a PL-map from an orientable PL-manifold $M^{n+2}$ onto $B^{n}$ such that each point preimage is collapsible to a 2-complex which is homotopy equivalent to $F^{2}$, a closed orientable surface of genus at least 2 , then $p$ can be approximated by a locally trivial bundle.

\section{REFERENCES}

1. G. Allaud and E. Fadell, A fiber homotopy extension theorem, Trans. Amer. Math. Soc. 104 (1962), 239-251.

2. D. R. Anderson, The Whitehead torsion of a fiber homotopy equivalence, Michigan Math. J. 21 (1974), 171-180.

3. J. Cerf, La stratification naturelle des espaces de fonctions differentiables reeles et le théorème de la pseudo-isotopie, Inst. Hautes Études Sci. Publ. Math. 39 (1970). 
4. T. A. Chapman, Lectures on Hilbert cube manifolds, CBMS Regional Conf. Ser. in Math., no. 28, Amer. Math. Soc., Providence, R. I., 1976.

5. __ Approximating maps into fiber bundles by homeomorphisms, Rocky Mountain J. Math. 10 (1980), 333-350.

6. __ Approximation results in topological manifolds, Mem. Amer. Math. Soc., vol. 34, no. $251,1981$.

7. T. A. Chapman and S. Ferry, Constructing approximate fibrations, Trans. Amer. Math. Soc. 276 (1983), 757-774.

8. D. Coram and P. Duvall, Approximate fibrations, Rocky Mountain J. Math. 7 (1977), 255288.

9. R. J. Daverman, Decompositions into codimension one submanifolds, Compositio Math. 55 (1985), 185-207.

10. __ Submanifold decompositions that induce approximate fibrations, Topology Appl. 33 (1989), 173-184.

11. __ PL maps with manifold fibers, preprint.

12. R. J. Daverman and J. J. Walsh, Decompositions into submanifolds that yield generalized manifolds, Topology Appl. 26 (1987), 143-162.

13. A. Dold and R. Lashof, Principal quasifibrations and fibre homotopy equivalence of bundles, Illinois J. Math. 3 (1959), 285-305.

14. D. B. A. Epstein, Curves on 2-manifolds and isotopies, Acta Math. 115 (1966), 83-107.

15. E. Fadell, On fiber homotopy equivalence, Duke Math. J. 26 (1959), 699-706.

16. F. T. Farrell, The obstruction to fibering a manifold over the circle, Bull. Amer. Math. Soc. 73 (1967), 737-740.

17. F. T. Farrell and W. C. Hsiang, H-cobordant manifolds are not necessarily homeomorphic, Bull. Amer. Math. Soc. 73 (1967), 741-744.

18. F. T. Farrell and L. E. Jones, Rigidity and other topological aspects of compact nonpositively curved manifolds, Bull. Amer. Math. Soc. (N.S.) 22 (1990), 59-64.

19. R. Goad, Approximate torus fibrations of high dimensional manifolds can be approximated by torus bundle projections, Trans. Amer. Math. Soc. 258 (1980), 87-97.

20. D. H. Gottlieb, A certain subgroup of the fundamental group, Amer. J. Math. 87 (1965), 840-856.

21. M. E. Hamstrom, The space of homeomorphisms on a torus, Illinois J. Math. 9 (1965), 59-65.

22. __ Homotopy groups of the space of homeomorphisms on a 2-manifold, Illinois J. Math. 10 (1966), 563-573.

23. L. S. Husch, Approximating approximate fibrations by fibrations, Canad. J. Math. 29 (1977), 897-913.

24. R. C. Kirby and L. C. Siebenmann, Foundational essays on topological manifolds, smoothings and triangulations, Ann. of Math. Stud., no. 88, Princeton Univ. Press, Princeton, N. J., 1977.

25. F. Laudenbach, Sur les 2-sphères d'une variéte de dimension 3, Ann. of Math. (2) 97 (1973), 57-81.

26. S. Mardesic and J. Segal, Shape theory, North-Holland, Amsterdam, 1982.

27. F. Quinn, Isotopy of 4-manifolds, J. Differential Geom. 24 (1986), 343-372.

28. L. C. Siebenmann, A total Whitehead torsion obstruction to fibering over the circle, Comment. Math. Helv. 45 (1970), 1-48.

29. E. H. Spanier, Algebraic topology, McGraw-Hill, New York, 1966.

30. J. Stasheff, A classification theorem for fiber spaces, Topology 2 (1963), 239-246.

31. N. Steenrod, The topology of fibre bundles, Princeton Univ. Press, Princeton, N. J., 1974.

32. F. Waldhausen, On irreducible 3-manifolds which are sufficiently large, Ann. of Math. (2) 87 (1968), 56-88. 
33. C. T. C. Wall, Surgery on compact manifolds, Academic Press, New York, 1970.

34. H. Zieschang, E. Vogt and H. D. Coldeway, Surfaces and planar discontinuous group, Lecture Notes in Math., vol. 835, Springer, Berlin, 1980.

Department of Mathematics, University of Tennessee, Knoxville, Tennessee 379961300

Current address: Department of Mathematics, Pusan National University, Pusan, Korea 60?735 